\title{
Mid- to Long-term Outcomes After Split-thickness Skin Graft vs. Skin Extension by Multiple Incisions
}

\author{
JETTE NICOLINE KERN ${ }^{1,2}$, FRIEDERIKE WEIDEMANN ${ }^{2}$, PADHRAIG F. O'LOUGHLIN ${ }^{3}$, \\ CHRISTIAN KRETTEK ${ }^{2}$ and RALPH GAULKE ${ }^{1,2}$ \\ ${ }^{1}$ Section Upper Extremity, Foot- and Rheuma Surgery, Trauma Department, \\ Medical School Hanover (MHH), Hanover, Germany; \\ ${ }^{2}$ Trauma Department, Medical School Hanover (MHH), Hanover, Germany; \\ ${ }^{3}$ Mater Hospital, City Gate Mahon, Cork, Ireland
}

\begin{abstract}
Background/Aim: Skin extension by multiple incisions (SEMI) may be superior to split-thickness skin graft (STSG) for closure of large soft tissue defects. Materials and Methods: Twenty-six patients who had undergone STSG were compared to 29 patients who had undergone SEMI on the extremities. Patient and Observer Scar Assessment Scale (POSAS), Dermatology Life Quality Index, Wound QoL (Quality of Life) and Short Form Health Survey 36 were used. Elasticity, thickness and skin sensation were compared between the treated and contralateral extremity. Range of motion in adjacent joints was measured. Complication rates were compared. Results: A total of 55 patients with a mean follow-up of 5.5 years (range $=2-9$ years) were examined. Patients with STSG had significantly worse scores in POSAS. The scar was thinner, less elastic and did not provide intact sensibility. Other scores, ROM and complication rates did not differ significantly. Conclusion: SEMI was superior to STSG regarding patient satisfaction and scar quality.
\end{abstract}

The coverage of large soft tissue defects that cannot be closed by direct suturing is challenging. Different techniques such as local and free flaps, and split-thickness skin graft (STSG) have been described. STSG need a vital wound bed to facilitate graft nutrition. STSG makes it possible to cover deep wounds from skin donor sites of a smaller size $(1,2)$.

This article is freely accessible online.

Correspondence to: Professor Dr. med Ralph Gaulke, Section Upper Extremity, Foot- and Rheuma Surgery, Trauma Department, Medical School Hanover (MHH), Carl-Neuberg-Str. 1, D-30625 Hannover, Germany. Tel: +49 5115322015, e-mail: gaulke.ralph@mh-hannover.de

Key Words: Split-thickness skin graft, skin extension by multiple incisions, SEMI, skin transplantation, skin quality.
However, disadvantages of STSG include unsightly scars, numbness, limited mobility and minor resistance to shearing forces. Shrinking of the graft may lead to joint contracture. The graft may appear thin and atrophic with an increased risk of disruption (3-7).

The human dermis consists of collagen fibres that prevent the skin from tearing, via their tensile strength and accompanying elastic fibres that return the skin to its original form once the stress force is gone. Many surgeons have made use of different skin components to develop new methods of delayed wound closure, in order to address the disadvantages of STSG. Subcutaneous expanders or traction devices use the robust nature of skin and its inherent elasticity to ultimately enable secondary wound closure by direct suturing. Unfortunately, such techniques have their own risks and are limited to relatively small soft tissue defects. Therefore, STSG remains the most frequently employed technique.

At the current authors' university-affiliated level one trauma centre, the technique of "Skin Extension by Multiple Incisions" (SEMI) (8) has been routinely employed to cover soft tissue defects since 2007. This technique is very easy to learn, leaves no donor site defect, covers the wound with fullthickness skin and leaves cosmetically-acceptable scars. Skin tension is reduced by multiple incisions of the dermis perpendicular to the skin tension lines, leaving the hypodermis intact. Skin sensitivity and mobility over the deep tissues are preserved and scar contractures have not occurred to date. These findings suggest that scars resulting from SEMI are more favourable than those resulting from STSG.

The aim of this retrospective study was to compare the clinical outcomes of STSG and SEMI.

\section{Patients and Methods}

Patients. All patients treated by STSG or SEMI at the extremities, between 2009 and 2015, at the current investigators' Universityaffiliated Level 1 Trauma Centre, were identified. Patients less than 
18 years of age, at the time of surgery, patients on immunosuppressive medication and wounds located on amputation stumps, torso or head were excluded. Further exclusion criteria included amputation of the treated or contralateral extremity, manipulation of the wound or scar after the primary operation, the application of a single technique to both extremities, other types of skin grafts performed on the contralateral extremity, instances where both STSG and SEMI were combined, a past history of mental illnesses, severe physical limitations, failure of the graft and death (Figure 1).

From August 2017 to April 2018, those patients who satisfied the inclusion criteria were studied with a minimum follow-up of 24 months.

Surgical technique of split-thickness skin grafting (STSG). A splitthickness skin graft, of 0.3 to $0.4 \mathrm{~mm}$ thickness was harvested from the donor site using an electric dermatome (9). The grafts were fenestrated to a mesh with an expanding ratio of 1:1.5 $(10,11)$ in order to increase graft surface area and size and to allow sufficient wound drainage. The graft was fixed with skin staples and covered with a vacuum seal to improve the contact of graft to the wound bed for five to seven days, depending on the vascular health of the wound base.

Surgical technique of skin extension by multiple incisions (SEMI). After wound closure, the surrounding skin tension was decreased via multiple dermal incisions perpendicular to the skin tension lines. The incisions were approximately 3 to $5 \mathrm{~mm}$ long (Figure 2). At the end of the surgery, the wounds are covered with a petrolatumimpregnated gauze and a compress dressing. It is possible to reverse the sequence of steps in order to approximate wound margins prior to suturing. A net-like full-thickness skin layer covering an intact subcutaneous flap is created in this way. Incising the elastic collagen fibres of the skin reduces the tension at the wound edges very effectively. Multiple incisions also allow wound fluid to drain and thus postoperative swelling is reduced.

In contrast to STSG, normal sensation and hair growth are preserved due to the presence of all skin layers. The colour of the operated area matches the surrounding skin aside from the multiple small white scars resulting from the small incisions. These small scars do not seem to bother the patients from a cosmetic perspective and in male patients, they are typically masked by hair growth.

\section{Outcome parameters}

Questionnaires. Four questionnaires were utilised to test the patient's subjective opinion regarding the postoperative result. Those sections of the questionnaires that were based on the results of the examination were completed by a single observer (JK), who was not involved directly in the patient's treatment.

The Patient and Observer Scar Assessment Scale (POSAS), first published in 2004 and updated in 2005 (12), consists of two parts: The Patient Scar Assessment Scale (PSAS) and the Observer Scar Assessment Scale (OSAS). It is the first questionnaire to consider the patients' subjective opinions of their scars. The patients assess their scar with regard to pain and itchiness in the previous week and scores colour, stiffness, thickness and irregularity. The observer evaluates vascularity, pigmentation, thickness, relief, pliability and the surface area of the scar. Different categories are available for each element, for further documentation aside from the scale. All criteria are compared to intact skin and scored on a ten-point-scale with one point indicating the best score and ten points reflecting the worst score. The six items respectively are combined to generate a total score ranging from 6 to 60 . Furthermore, an overall impression is recorded. The POSAS is regarded as a reliable and valid scar assessment tool (13).

The Questionnaire on Quality of Life with chronic wounds (short Wound-QoL=W-QoL) focuses on the impact of a chronic wound on the health-related quality of life of patients (14). It was developed from three established disease-specific wound questionnaires (Freiburg Quality of Life Assessment for Wounds (15), Cardiff Wound Impact Schedule (16) and Würzburg Wound Score (17)) with the goal of developing a consolidated, simple and short questionnaire (14). The short Wound-QoL consists of 17 items relating to potential impairment caused by a wound in the previous seven days. These items are scored on a five-point-scale from 'not at all' to 'very', with responses coded with numbers from zero to four. A global score is calculated by averaging all items. Furthermore, three subs-scores, relating to 'Body' (Item 1 to 5), 'Psyche' (Items 6 to 10) and 'Everyday life' (Items 11 to 16) can be generated. Currently, it is considered to be more reliable and valid than the original instruments (18).

The Dermatology Life Quality Index (DLQI) was developed to measure the impact of skin conditions on the patients' life (19). The questionnaire consists of 10 questions. They refer to symptoms and feelings, daily activities, leisure, work or school, personal relationships and side-effects of treatment. The questions are answered by the patient with four alternative responses (19) ranging from 0 to 3 points ('Not at all' to 'Very much'). The response 'Not relevant' is also scored as zero points. A total score is calculated with a range from 0 to 30 . A high score indicates a large impact on a patient's life (20). Furthermore, subscales can be generated. The DLQI can be used for any skin condition and has been shown to be a reliable indicator of life quality for several skin diseases $(19,21)$. It has already been used but has not yet been validated, in research on scars $(21,22)$.

The Short-Form 36 questionnaire (SF-36) was developed from the Medical Outcome Study (MOS). It was created to measure health levels in any patient or population group (23). It is used in clinical practice and research, as well as in general population surveys. The SF-36 consists of 36 items unequally subdivided into eight categories: physical functioning, role limitation due to physical problems, pain, social functioning, mental health, role limitation due to emotional problems, vitality and general health $(23,24)$. All questions refer to the previous four weeks. Low scores represent bad health and high scores represent preferable health (24).

Clinical examination. Clinical examination included sensation testing with the Sharp-Blunt-Distinction and the Two-PointThreshold (2-PT) test. The Sharp-Blunt-Distinction test was performed using both ends of a cotton bud with one sharp and one blunt end. Two-point-threshold (2-PT) was measured with a twopoint touch discriminator (AREX ${ }^{\circledR}$, Palaiseau, France). Distances between two spines were standardized at 2, 3, 4, 6, 8, 10, 15, 20 or $25 \mathrm{~mm}$. Patients were asked whether they experienced "one" or "two" sensations when touched with two spines. The smallest distance the patient recognizes as two points represents the 2-pointthreshold. In sensation testing, patients were scored with 2 points if they passed the test on both extremities, meaning that they distinguished sharp from blunt touches or detected two separate points, 1 point if they passed the test on one extremity only and 0 points if they did not pass the test at all. 


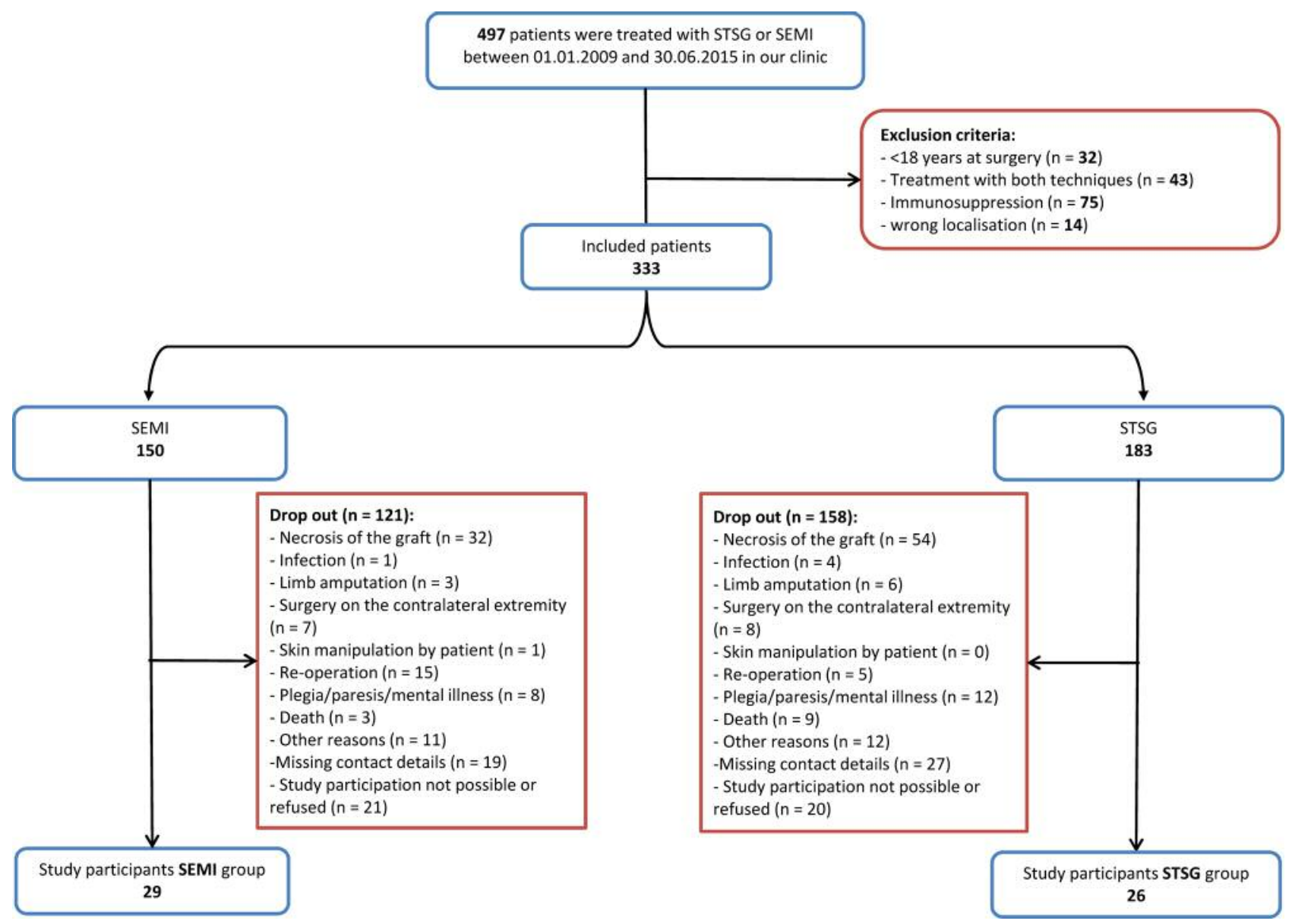

Figure 1. Patients collection.

Range of motion (ROM) of joints next to the graft were measured using a goniometer.

Skin thickness was measured with a $40 \mathrm{~Hz}$ ultrasound L18-5 transducer on an Affiniti $70 \mathrm{G}$ (Philips Healthcare, Hamburg, Germany). Gel was used as the conductive medium.

Skin elasticity and related parameters were measured with the non-invasive Cutometer ${ }^{\circledR}$ MPA 580 dual (Courage + Khazaka, Cologne, Germany). A hand-held probe is connected to a device that generates negative pressure. This sucks skin into the probe. Inside the probe, an optical system measures the elongation of skin. The Cutometer can be used with a 2-, 4-, 6- or 8-mm, diameter probe. In this study, a probe with a diameter of 6-mm was used which is recommended to measure the mechanical properties of the dermis. A wider probe diameter measures deeper soft tissue layers for elasticity. There are four different modes of pressure application. In the time-strain-mode (Mode 1), which was used in this study, a constant negative pressure is applied and released promptly. The recorded curve (Figure 3 ) shows the skin deformation $(\mathrm{mm})$ as a function of time (s). Each measurement cycle consists of two phases: 1. the suction phase and 2. the retraction phase. The rising part of the curve represents the suction phase with skin drawn into the probe. As the negative pressure drops, the retraction phase starts and the curve decreases. The amount of negative pressure and the length of phases can be adjusted manually. In the current study, a negative pressure of 450 mbar was applied over $2 \mathrm{sec}$ with repetition of three cycles per measured skin area. If the same skin area is tested multiple times its mechanical properties change due to residual deformation. This phenomenon, called tissue fatigue, can be seen as the maximal distension increase during multiple measurement cycles.

The maximal depth of skin drawn into the probe in the first measurement cycle, also called final distension, is described as R0 (Figure 3). As the negative pressure ceases, the skin retracts to its baseline position. The total recovery after the suction phase is described as R8.

Testing of sensibility and measuring of skin thickness and skin elasticity were performed on nine localisations on each site (Figure 4): First measurement was taken at the centre of the scar, marked as point 1 in the diagram. $\mathrm{x}$ and $\mathrm{y}$ describe the distance between the centre and proximal and lateral margins of the scar, respectively. Points 2 to 5 were located perifocal (medial, proximal, lateral and distal) halfway between centre and margins of the scar $(=1 / 2 \times$ and $1 / 2$ y). Points 6 to 9 were measured approximately $2 \mathrm{~cm}$ outside the scar. All nine points were measured on the opposite site for 


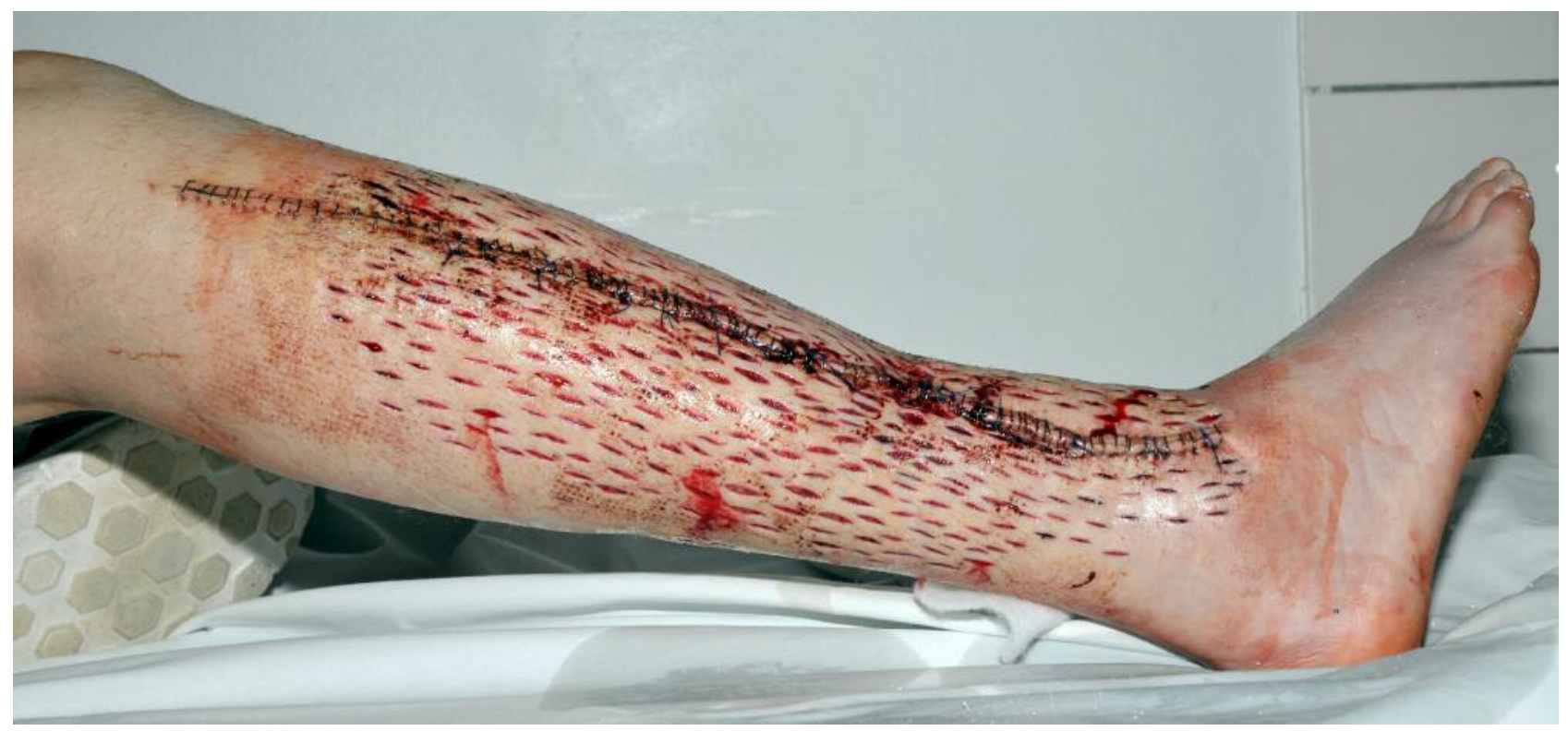

Figure 2. Skin extension by multiple incisions on lower leg day one post-secondary wound closure following compartment dissection whilst treating a lower leg fracture.

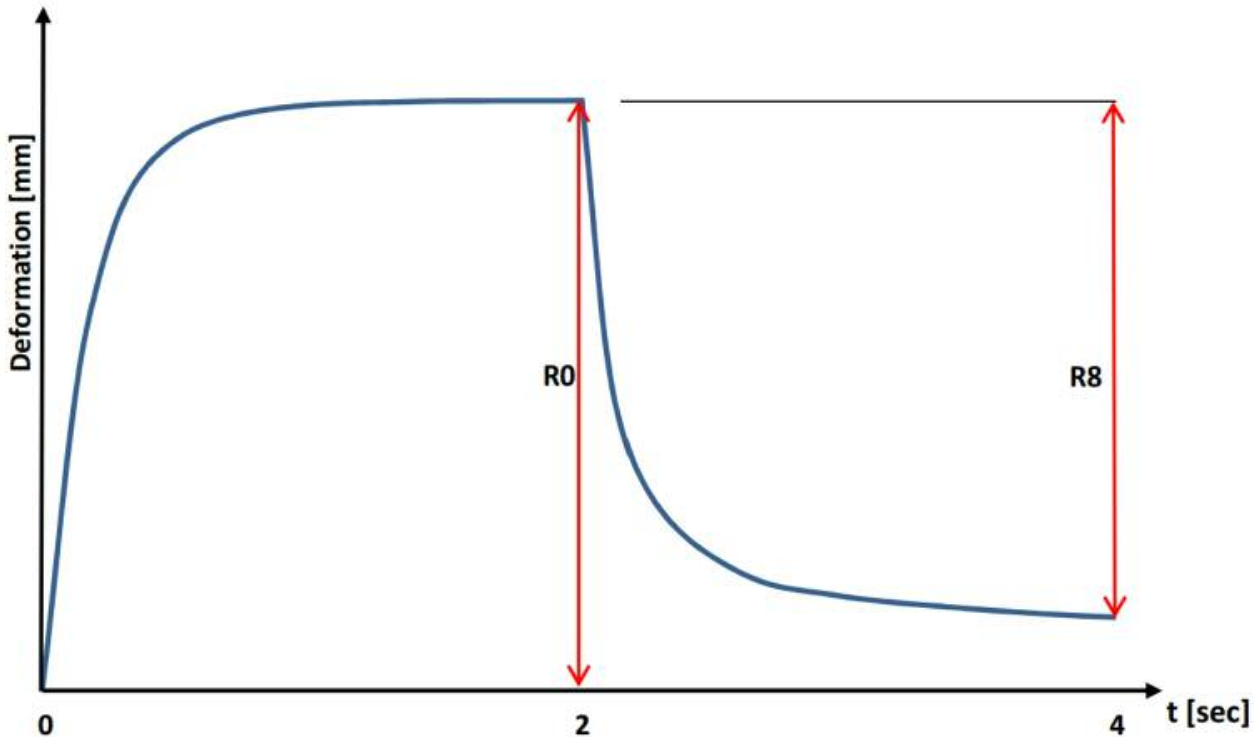

Figure 3. Cutometer measurement cycle.

comparison, summing up to 18 values per tested item (elasticity, thickness and sensibility) per patient.

Statistical analysis. PASW Statistics $18^{\circledR}$ (SPSS Inc., Hong Kong) was used to perform Student's $t$-tests and alternatively Pearson's Chisquared tests if Kolmogorov-Smirnov-test did not reveal a normal distribution of data. Statistical significance was assumed if $p \leq 0.05$.

\section{Results}

Between 2009 and 2015, 497 patients received STSG or SEMI. A total of 55 patients were examined in the current study. Twenty-six patients received a STSG and 29 patients a SEMI (Figure 1). 


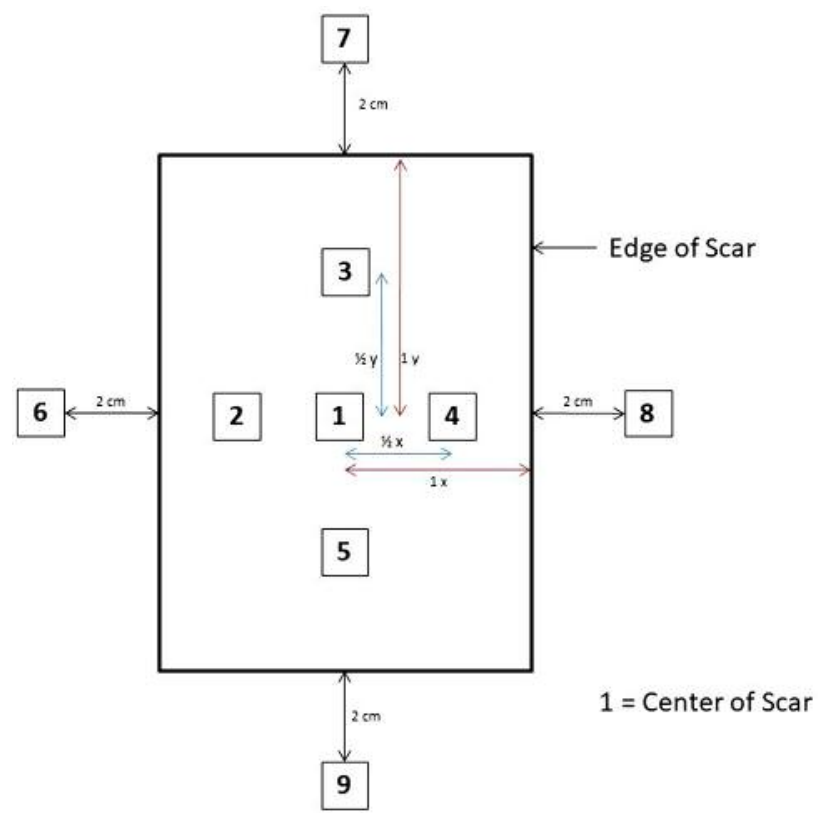

Figure 4. Measurement locations.

At surgery, patients of the SEMI group were younger compared to those of the STSG group (48 years, range $=22-76$ years, $v s .52$ years, range $=20-86$ years; $p=0.4516)$. The same distribution was found at follow-up (53 years, range $=27-78$ years, $v s .58$ years, range $=24-92$ years; $p=0.2909$ ). Follow-up was significantly shorter in the SEMI group (59 months, range=27-104 months, vs. 74 months, range=33-104 months; $p=0.0288$ ).

Scars of the STSG group were significantly longer $(97 \mathrm{~mm}$, range $=10-165 \mathrm{~mm}, v s .155 \mathrm{~mm}$, range $=22-490 \mathrm{~mm} ; p=0.0085)$ while the width of scars did not differ $(81 \mathrm{~mm}$, range $=25-200$ $\mathrm{mm}, v s .78 \mathrm{~mm}$, range $=10-205 \mathrm{~mm} ; p=0.7809)$. The total scar size did not differ significantly $\left(89 \mathrm{~cm}^{2}\right.$, range $=3.8-270 \mathrm{~cm}^{2}$, vs. $135 \mathrm{~cm}^{2}$, range $\left.=2.2-539 \mathrm{~cm}^{2} ; p=0.1195\right)$.

Most of the STSG scars were located on the lower limb. SEMI scars were rarely found on the lateral aspect of the treated extremities (Table I).

Questionnaires. Patients of the SEMI group generated lower scores for all PSAS categories than patients in the STSG group. This indicated a better scar outcome. The differences between groups were significant with regard to thickness $(2.79 \pm 2.16$, range $=1-10, v s .5 .27 \pm 2.91$, range $1-10 ; p=0.0007)$, irregularity $(2.79 \pm 2.29$, range $=1-10, v s .5 .27 \pm 3.14$, range $1-10 ; p=0.0001)$ and overall opinion $(3.28 \pm 2.66$, range $1-10$, vs. $6.38 \pm 2.89$, range $=1-10 ; p=0.0001)$. Furthermore, the total score was significantly lower in the SEMI group $(16.79 \pm 9.26$, range=642 , vs. 23.92 \pm 11.15 , range $=6-41 ; p=0.0124)$. Differences in the categories of pain $(1.72 \pm 1.64$, range $=1-8, v s$. 2.04 \pm 2.50 , range $=1-10 ; p=0.6287)$, itchiness $(1.79 \pm 1.32$, range $=1-6, v s$. $1.84 \pm 2.24$, range $=1-9 ; p=0.9247)$ and colour $(4.62 \pm 3.02$, range $=1-10, v s .5 .00 \pm 2.74$, range $=1-10 ; p=0.6287$ ) were not significant. A trend towards significance was observed when comparing pliability with scores of $3.17 \pm 2.79$ (range=1-10) vs. $4.56 \pm 2.63$ (range $=1-10 ; p=0.0671$ ) (Table II).

The OSAS scores, representing the observers' opinion, were significantly lower in the SEMI group for all sub-categories, the overall impression and the total score (Table II).

The mean score of the category 'overall opinion' was higher in the PSAS compared to the OSAS (SEMI: 3.28 points in PSAS $v s .2 .28$ points in OSAS; STSG: 6.38 points in PSAS $v s .4 .54$ points in OSAS; $p=0.2728)$.

SEMI patients scored fewer points in the total score of the DLQI than STSG patients $(2.14 \pm 3.69$, range $=0-13$, vs . $4.42 \pm 6.32$, range $=0-20 ; p=0.1146)$. This questionnaire assesses the impact on a patient's daily life caused by a scar. Lower values represent less impairment. The difference between groups was not statistically significant ( $p=0.1146)$.

Performing Pearson's Chi-Square test for single questions of the DLQI, significance was established with regard to question 1, 'Over the last week, how itchy, sore, painful or stinging has your skin been?' ( $p=0.0141)$, and question 4, 'Over the last week, how much has your skin influenced the clothes you wear?' $(p=0.0371)$. The DLQI total score correlates significantly with the PSAS overall impression $(p<0.0001, \mathrm{r}=0.5612)$.

In the W-QoL questionnaire, the median of scored points, in both groups, for all items was 0 points except for the question 'In the last seven days, I was afraid knocking the wound'. In this question the SEMI group's median was 0 points while STSG group's median was 1 point. No significant differences were found between the SEMI and STSG group with regard to the total score $(0.44$, range $=0.00$ 2.12 , vs. 0.71 , range $0.00-3.35 ; p=0.2424)$, or the subscales 'body' (0.29, range=0.00-1.60, vs. 0.38, range 0.00-2.40; $p=0.5741)$, and 'everyday life' $(0.60$, range $=0.00-3.33, v s$. 0.70 , range $=0.00-3.83 ; p=0.7251)$. A significant difference was found in the subscale 'psyche' $(0.46$, range $=0.00-2.40$, vs. 1.14 , range $=0.003 .80 ; p=0.0253$ ).

SF-36 results revealed no significant differences between the groups. Patients in the SEMI group scored fewer points in all categories except for 'Energy and Fatigue' where both groups scored 56 points. For 'Physical Functioning', SEMI patients scored 65 points and STSG patients scored 70 points. Regarding 'Role limitation due to physical health' groups scored 65 and 70 points; 'Role limitation due to emotional problems' 68 and 79 points; 'Emotional wellbeing' 71 and 73 points; 'Social functioning' 76 and 81 points; 'Pain' 60 and 78 points and 'General Health' 60 and 63 points, respectively. Standard deviations displayed a wide range from 19 to 46 points. 
Table I. Demographic characteristics.

\begin{tabular}{lcc}
\hline & SEMI & STSG \\
\hline Number of patients & 29 & 26 \\
Female/Male & $22 / 7$ & $52(44-59 ; 19)$ \\
Mean age at surgery, years (95\%CI; SD) & $48(43-54 ; 15)$ & $58(51-65 ; 18)$ \\
Mean age at follow up, years (95\%CI; SD) & $53(47-59 ; 15)$ & $74(64-83 ; 23)$ \\
Follow-up, month (95\%CI; SD) & $59(50-69 ; 25)$ & \\
Scar & & $134.53,83.2-185.84 ; 127.06$ \\
Total scar size, cm ${ }^{2}$ (mean, 95\%CI; SD) & $89.07,60.38-117.76 ; 72.51$ & $77.88,58-98 ; 49.26$ \\
Width, mm (mean, 95\%CI; SD) & $81.44,64-99 ; 43.4$ & $154.88,114-195,100.3$ \\
Lenght, mm (mean, 95\%CI; SD) & $97.37,81-114 ; 42.2$ & 15 \\
Localisation & & 11 \\
Right & 17 & 4 \\
Left & 12 & 1 \\
Lower arm & 6 & 2 \\
Wrist & 6 & 1 \\
Upper limb & 1 & 14 \\
Knee & 0 & 2 \\
Lower limb & 3 & 2 \\
Ankle & 9 & 5 \\
Foot & 4 & 7 \\
Joint involved & 15 & 4 \\
Lateral & 2 & 7 \\
Medial & 5 & 7 \\
Extension site & 13 & 1 \\
Flexion site & 8 & \\
Other & 1 & \\
\hline
\end{tabular}

Clinical examination. Sensitivity to touch differed significantly between study groups in the Sharp-BluntDistinction test, within the scar margins compared to the control site (measurement points $1-5 ; p<0.0001$ ). In the Sharp-Blunt-Distinction test, most patients of the SEMI group scored 2 points within the scar area on measurement points 1-5. This was true, on average, for 25 patients of 29 patients in this group. In the STSG group, 24 of 26 patients scored 1 point in this area while 2 patients did not score a point. This means most STSG patients passed the test on the non-operated site but failed for the grafted area, while almost all SEMI patients passed the test on both sides. For the adjacent skin (measurement points 6-9) almost all SEMI patients passed the test (average 25 of 29 patients). With these measurement points several STSG patients scored 2 points, while 6 STSG patients did not demonstrate wholly intact sensation and scored only 1 point. 2 patients scored 0 points. In total, 2 patients from each group were unable to feel any sensation due to an underlying polyneuropathy secondary to diabetes or other causes.

The 2-Point-Threshold test revealed that 19 of 27 tested patients $(70 \%)$ in the SEMI group had intact sensation in the centre of the flap and on the contralateral site. For the adjacent measurement points (MP2-5) 16-17 of 27 (59-63\%) patients scored 2 points (Table III). In the same area (MP1-5) 6-9
Table II. POSAS scores.

\begin{tabular}{lccc}
\hline & SEMI & STSG & $p$-Value \\
\hline PSAS category & & & \\
Pain & 1.72 & 2.00 & 0.6287 \\
Itching & 1.79 & 1.84 & 0.9247 \\
Colour & 4.62 & 5.00 & 0.6287 \\
Pliability & 3.17 & 4.56 & 0.0671 \\
Thickness & 2.79 & 5.27 & 0.0007 \\
Irregularity & 2.79 & 5.97 & 0.0001 \\
Total score & 16.79 & 23.92 & 0.0124 \\
Overall opinion & 3.28 & 6.38 & 0.0001 \\
OSAS category & & & \\
Vascularity & 1.72 & 3.04 & 0.0027 \\
Pigmentation & 2.83 & 4.35 & 0.0036 \\
Thickness & 2.00 & 4.38 & 0.0000 \\
Relief & 2.62 & 3.96 & 0.0020 \\
Pliability & 1.93 & 4.35 & 0.0000 \\
Surface area & 1.55 & 3.92 & 0.0000 \\
Total score & 12.66 & 24.00 & 0.0000 \\
Overall opinion & 2.28 & 4.54 & 0.0000 \\
\hline
\end{tabular}

patients scored one point (22-33\%) while 2-3 patients scored 0 points. Patients in the STSG group scored either 0 points or 1 point within the scar area (measurement points 1-5). One patient was able to pass the 2-PT-test on one point located in 
Table III. Two-point-discrimination.

\begin{tabular}{|c|c|c|c|c|c|c|}
\hline & \multicolumn{3}{|c|}{ SEMI (n=27) } & \multicolumn{3}{|c|}{ STSG $(n=25)$} \\
\hline & $\begin{array}{l}\text { Intact sensation } \\
\text { on both sites }\end{array}$ & $\begin{array}{l}\text { Intact sensation } \\
\text { on one site }\end{array}$ & $\begin{array}{c}\text { No } \\
\text { sensation }\end{array}$ & $\begin{array}{c}\text { Intact sensation } \\
\text { on both sites }\end{array}$ & $\begin{array}{l}\text { Intact sensation } \\
\text { on one site }\end{array}$ & $\begin{array}{c}\text { No } \\
\text { sensation }\end{array}$ \\
\hline Scar area & $17(63 \%)$ & $7(26 \%)$ & $3(11 \%)$ & $0(0 \%)$ & $18(72 \%)$ & $7(28 \%)$ \\
\hline Centre (MP1) & $19(70 \%)$ & $6(22 \%)$ & $2(7 \%)$ & $0(0 \%)$ & $18(72 \%)$ & $7(28 \%)$ \\
\hline MP 2 & $17(63 \%)$ & $7(26 \%)$ & $3(11 \%)$ & $0(0 \%)$ & $20(80 \%)$ & $5(20 \%)$ \\
\hline MP 3 & $16(59 \%)$ & $8(30 \%)$ & $3(11 \%)$ & $1(4 \%)$ & $18(72 \%)$ & $6(24 \%)$ \\
\hline MP 4 & $17(63 \%)$ & $6(22 \%)$ & $4(15 \%)$ & $0(0 \%)$ & $18(72 \%)$ & $7(28 \%)$ \\
\hline MP 5 & $16(59 \%)$ & $9(33 \%)$ & $2(7 \%)$ & $0(0 \%)$ & $14(56 \%)$ & $11(44 \%)$ \\
\hline Adjacent skin & $18(67 \%)$ & $6(22 \%)$ & $3(11 \%)$ & $10(40 \%)$ & $9(36 \%)$ & $6(24 \%)$ \\
\hline MP 6 & $19(70 \%)$ & $6(22 \%)$ & $2(7 \%)$ & $8(32 \%)$ & $11(44 \%)$ & $6(24 \%)$ \\
\hline MP 7 & $21(78 \%)$ & $3(11 \%)$ & $3(11 \%)$ & $12(48 \%)$ & $7(28 \%)$ & $6(24 \%)$ \\
\hline MP 8 & $21(78 \%)$ & $3(11 \%)$ & $3(11 \%)$ & $10(40 \%)$ & $8(32 \%)$ & $7(28 \%)$ \\
\hline MP 9 & $17(63 \%)$ & $8(30 \%)$ & $2(7 \%)$ & $8(32 \%)$ & $12(48 \%)$ & $5(20 \%)$ \\
\hline
\end{tabular}

Table IV. Mean skin thickness.

\begin{tabular}{|c|c|c|c|c|c|c|c|c|c|}
\hline & \multicolumn{5}{|c|}{ SEMI (n=17) } & \multicolumn{3}{|c|}{ STSG $(n=17)$} & \multirow{2}{*}{ 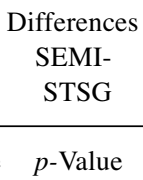 } \\
\hline & Ipsilateral & Contralateral & Diff. & $p$-Value & Ipsilateral & Contralateral & Diff. & $p$-Value & \\
\hline Scar area $(95 \% \mathrm{CI})$ & $0.90(0.74-1.05)$ & $0.87(0.71-1.04)$ & 0.023 & 0.2802 & $0.86(0.67-1.06)$ & $1.06(0.78-1.33)$ & -0.194 & 0.0296 & 0.0188 \\
\hline Adjacent skin & $0.90(0.71-1.09)$ & $0.90(0.72-1.07)$ & 0.003 & 0.9169 & $1.16(0.86-1.45)$ & $1.04(0.77-1.30)$ & 0.119 & 0.1105 & 0.1289 \\
\hline
\end{tabular}

the graft area. On the adjacent healthy skin only between 8 and 12 of 25 STSG patients (32-48\%) demonstrated intact sensation for both tested sites while the other STSG patients scored only 1 or 0 points (Table III).

Range of motion was measured for the joint most affected by the wound closure technique and the joints proximal and distal to the scar. If the scar was located between two joints then the ROM was measured for these two joints. Differences in ROM were typically attributable to posttraumatic articular changes rather than scar contracture. However, these observed differences were not statistically significant. Eight patients underwent arthrodesis and therefore were excluded from the study.

Skin thickness was measured for 17 patients of each group. In the SEMI group, within the scar area, measurement points 1 to 5 , average skin thickness was $0.90 \mathrm{~mm}$ $(95 \% \mathrm{CI}=0.74-1.05)$ on the treated site and $0.87 \mathrm{~mm}$ (95\% CI $=0.71-1.04)$ on the control site. The difference of $0.023 \mathrm{~mm}$ was not significant $(p=0.2802)$. In the same area, in the STSG group, skin thickness was $0.86 \mathrm{~mm}$ $(95 \% \mathrm{CI}=0.67-1.06)$ on the treated site and $1.06 \mathrm{~mm}$
(95\% CI $=0.78-1.33)$ on the control site. The difference of $0.194 \mathrm{~mm}$ was significant $(p=0.0296)$. A comparison between skin thickness differences between the groups revealed a significant difference within the scar area (SEMI $0.023 \mathrm{~mm}, 95 \% \mathrm{CI}=0.022-0.068$, range $=0.21-0.12$, vs. STSG $-0.194 \mathrm{~mm}, \quad 95 \% \mathrm{CI}=0.366-0.022, \quad$ range $=0.91-0.16$; $p=0.0188$ ) while in the adjacent skin the difference between groups was not significant (SEMI $0.003 \mathrm{~mm}, 95 \% \mathrm{CI}=0.054-$ 0.060 , range $=0.19-0.26$, vs. STSG $0.119 \mathrm{~mm}, 95 \% \mathrm{CI}=0.031-$ 0.27 , range $=0.11-1.17 ; p=0.1289)$ (Table IV).

Results of skin elasticity measurements using a Cutometer ${ }^{\circledR}$ MPA 580 did not show significant differences with regard to parameters R0, R2 or R8 within the scar edges. The average of the differences between operated and non-operated sites was compared. The average difference of skin firmness in the centre of the scar (measurement point 1) was $-0.09 \pm 0.29$ $(95 \% \mathrm{CI}=-0.20-0.03$, range $=-0.94-0.40) \mathrm{mm}$ in SEMI group and $0.25 \pm 0.40(95 \% \mathrm{CI}=-0.42--0.09$, range $=-1.56-0.42) \mathrm{mm}$ in the STSG group. However, although the difference in the SEMI group was smaller compared to the STSG group, it is not significant $(p=0.1121)$. The average bilateral difference of 
Table V. Cutometer results absolute data.

\begin{tabular}{|c|c|c|c|c|}
\hline Raw Data & Localisation/measurement point (MP) & SEMI & STSG & $p$-Value \\
\hline \multirow{4}{*}{$\begin{array}{l}\text { Ipsilateral } \\
\text { (operated site) }\end{array}$} & Scar centre (MP 1) (range, 95\%CI) & $1.29(0.54-2.00,1.15-1.45)$ & $0.98(0.50-1.71,0.82-1.14)$ & 0.0044 \\
\hline & Average pericentral (MP 2-5) (range, 95\%CI) & $1.28(0.57-1.99,1.15-1.42)$ & $1.03(0.52-1.86,0.88-1.18)$ & 0.0128 \\
\hline & Scar area (MP 1-5) (range, $95 \% \mathrm{CI})$ & $1.29(0.57-1.94,1.15-1.42)$ & $1.02(0.53-1.83,0.87-1.17)$ & 0.0092 \\
\hline & Average adjacent skin (MP 6-9) (range, $95 \% \mathrm{CI}$ ) & $1.23(0.60-1.75,1.13-1.34)$ & $1.20(0.71-2.01,1.07-1.33)$ & 0.5201 \\
\hline \multirow{4}{*}{$\begin{array}{l}\text { Contralateral } \\
\text { (control site) }\end{array}$} & Centre contralateral (MP 1) (range, $95 \% \mathrm{CI})$ & $1.39(0.61-2.10,1.24-1.54)$ & $1.25(0.64-2.11,1.07-1.37)$ & 0.1219 \\
\hline & Average pericentral (MP 2-5) (range, 95\%CI) & $1.36(0.59-2.01,1.23-1.49)$ & $1.25(0.66-2.00,1.12-1.39)$ & 0.2519 \\
\hline & Contralateral MP 1-5 (range, $95 \% \mathrm{CI})$ & $1.36(0.6-2.03,1.23-1.50)$ & $1.25(0.66-2.02,1.11-1.38)$ & 0.2115 \\
\hline & Average adjacent skin (MP 6-9) (range, 95\%CI) & $1.31(0.73-1.80,1.20-1.42)$ & $1.22(0.62-1.91,1.08-1.35)$ & 0.1531 \\
\hline
\end{tabular}

Table VI. Complication rates.

\begin{tabular}{|c|c|c|c|}
\hline & Total numbers (patients) & SEMI procedures & STSG procedures \\
\hline & $679(498)$ & 279 & 400 \\
\hline \multicolumn{4}{|l|}{ Procedures } \\
\hline Exclusion criteria $<18$ years at operation & 41 & 12 & 29 \\
\hline Wrong localisation* & 15 & 2 & 13 \\
\hline Immunosuppression & 104 & 33 & 71 \\
\hline Included procedures & $519(377)$ & 232 & 287 \\
\hline Complications $* *$ & 159 & 60 & 99 \\
\hline Premature termination $* * *$ & 5 & 3 & 2 \\
\hline Infections & 6 & 1 & 5 \\
\hline No complications & 349 & 167 & 182 \\
\hline
\end{tabular}

All performed SEMI and STSG procedures from 01.01.2009 to 30.06.2015 in Hannover Medical School in the Department of trauma surgery. *Wound located on amputation stumps, torso or head; **Complications: necrosis, impaired wound healing, wound dehiscence, secondary granulation;

***Premature termination: manipulation of wound and scar, premature stitch removal, re-operation.

measurement points 2 to 5 (pericentral inside scar margins) was not significant between SEMI and STSG $(-0.58 \pm 0.21 \mathrm{~mm}$, $95 \% \mathrm{CI}=-0.14-0.02$, range $=-0.38-0.57, v s .-0.22 \pm 0.44 \mathrm{~mm}$, $95 \% \mathrm{CI}=-0.41-0.03$, range $=1.48-0.58 ; p=0.1077)$. Differences in skin firmness in the adjacent normal skin next to the scar were not significantly different (range $=-0.08 \pm 0.17 \mathrm{~mm}$, $95 \% \mathrm{CI}=0.14--0.01$, range $=-0.41-0.25, v s .-0.02 \pm 0.19 \mathrm{~mm}$, $95 \% \mathrm{CI}=-0.10-0.06$, range $=-0.54-0.25 ; p=0.2545)$.

Focusing on absolute values (Table V) of the measurement points on the treated site, which were not compared to the control site, a significant difference was found in the measured values for R0 within scar margins (measurement points 1 to 5 ) (SEMI $1.29 \mathrm{~mm}, 95 \% \mathrm{CI}=1.15-1.42$, range $=0.57-1.94$, vs. STSG $1.02 \mathrm{~mm}, 95 \% \mathrm{CI}=0.87-1.17$, range $=0.53-1.83$; $p=0.0092)$. The average of absolute data outside scar margins (points 6 to 9) did not differ significantly between groups (SEMI $1.23 \mathrm{~mm}, 95 \% \mathrm{CI}=1.13-1.34$, range $=0.60-1.75$, vs. STSG $1.20 \mathrm{~mm}, 95 \% \mathrm{CI}=1.07-1.33$, range=0.71-2.01; $p=0.5201)$. Absolute values on the non-operated site were not significantly different between groups (Table V).
One patient of the STSG group was not included in the analysis because he received a muscular flap under the STSG. The muscular flap is extra tissue in the operated location and therefore it influences the elasticity measurements.

Complication rates. A retrospective analysis was performed with regard to complications. From 01.01.2009 to 30.06.2015 679 SEMI and STSG procedures were performed in the authors' level 1 trauma department. There were 279 SEMI procedures and 400 STSG procedures. Overall, 519 procedures (232 SEMI and 287 STSG) met the inclusion criteria. Necrosis, disturbed wound healing, dehiscence of the wound and secondary granulation were summarized under the term 'complications' while infections were considered separately. There were five procedures which were prematurely terminated due to manipulation of wound and scar, premature stitch removal or re-operation in the same area. Pearson's Chi-Square test did not reveal any significant differences, in terms of complications, between the groups ( $p=0.067)$ (Table VI). 


\section{Discussion}

The aim of this retrospective study was to compare the clinical outcomes of STSG and SEMI and the study data showed that SEMI is superior to STSGs with regard to skin thickness, sensation, elasticity and a subjective assessment by patients and assessors.

Questionnaires. The results of POSAS showed superiority of SEMI over STSG in the patients' and the observer's scar assessment. It has been validated for use with any scar type $(25,26)$ which is important as scars resulting from SEMI look different to STSG scars. The current study data are very similar to those found by Busche et al. (27). They compared burn scars after conservative treatment, STSG alone and STSG in combination with Matriderm ${ }^{\circledR}$ consisting of bovine collagen using POSAS and Cutometer ${ }^{\circledR}$ MPA 580. Conservatively treated patients scored their scar lower with regard to skin thickness than patients treated with STSG or STSG+Matriderm ${ }^{\circledR}$ (3.6 vs. 5.04 vs. 5.17). In accordance with Busche et al. the current authors found that SEMI patients gave significantly less points in regard to skin thickness than STSG patients (2.79vs. 5.27) $(p<0.001)$. In Busche's study the PSAS total score was lower in the conservatively treated group compared to STSG and STSG+Matriderm ${ }^{\circledR}$ group (23.79 vs. 29.01 vs. 32.34; $p=0.72$ ). In the current study, the SEMI group gave an even lower total score whilst the STSG group scored similar to Busche's conservatively treated group (16.79 vs. 23.92; $p=0.0124)$. Draaijers et al. found itchiness and thickness to have the greatest influence on the patient's overall impression (12), which was confirmed by Busche et al. However, in the current study, scores for itchiness were low in both groups (SEMI 1.79, STSG 1.84). Other studies have reported itchiness in STSG scars as well $(28,29)$ but they referred on burn-scars. This patient group was excluded from the current study as per the study's protocol.

The observer's part of POSAS revealed highly significant differences between the groups. However, a conclusion should be drawn with caution as the observer was not blinded in this study, given how distinctive the respective scars look. This is the major limitation of our study. Still, from a clinician's point of view, SEMI scars had a better clinical outcome than STSG. The mean overall impression score was higher in the PSAS compared to the OSAS (SEMI: $3.28 v s .2 .28$ points and STSG: $6.38 v s .4 .54$ points, respectively). This indicates a worse perception of the scars by the patients (25).

The DLQI was developed for any skin disease, but is often used together with scar scales. It focuses on impairments in daily life caused by skin conditions. These impairments are not included in objective scar scales (22, 30). Reinholz et al. proved it to be useful in assessing quality of life in patients with different scar types (22). He found total scores of $2.07 \pm 3.56$ for physiologic scars (22) while regular psoriasis patients scored approximately 8.9 06.3 points (19). In the current study, the total scores in DLQI were low for both, SEMI $(2.14 \pm 3.69$, range=0-13) and STSG $(4.42 \pm 6.32$, range $=0-20)$. The authors observed that impairment is equal in regular scars and SEMI but higher in STSG. These differences are not significant $(p=0.1146)$.

The authors did not observe any significant differences in the average total score between study groups in the Questionnaire on Quality of Life with chronic wounds (WQoL). Also, two subscales did not differ significantly. As the minimum follow-up period was set at 24 months by the study protocol, mature scars and closed wounds were seen. Also, the questionnaire focused on chronic open wounds (31) so this finding is not surprising. The median score was 0.00 points in all items but one. This item was worded "In the last seven days, I was afraid of knocking the wound". This fear was present in many STSG patients because of the altered sensation and the relative fragility of the thin scar. STSG patients scored more points in this question than SEMI patients although no significance was reached. These results show that the W-QoL is not useful to measure the clinical outcome of closed wounds. The current study cohort was heterogeneous in respect to the trauma involved and the patients' economic as well as health status. Information was collected about the general health status using the questionnaire SF-36. Means and standard deviations ranged widely within the eight categories. These findings show that patients perceive their health status as either really good or really bad. From the authors' perspective the severity of the trauma sustained does not correlate with the general health status, indicating that patients cope differently. Many studies have used the SF-36 to assess patients' quality of life. It is mostly used for follow-up and assessment of change in patients' quality of life over time. Salomé et al. have used SF-36 on patients with chronic venous ulcers who received either STSG or conservative treatment. They found that SF36 improved significantly after STSG while values of the conservative treatment group did not improve or got even worse (32). In the current study cohort, patients completed the SF-36 questionnaire only at follow-up so a definitive conclusion cannot be drawn as to a difference between and within the groups over time.

Clinical examination. With the STSG technique, deep dermal structures and subcutaneous fat are not transferred to the soft tissue defect. The absence of these structures leads to reduced skin thickness, scarification between the wound base and the skin graft and insensitivity within the scar. As nerves are embedded in the dermis it is not surprising that STSG patients do not regain normal sensation. Using the SEMI 
technique, the elastic collagen fibres are cut by multiple incisions to reduce tension on the dermis. Cutaneous nerves are left intact and maintain normal skin sensation. Therefore, the current authors hypothesised a significant difference between the groups regarding scar area sensation. This hypothesis was proved to be true. Interestingly, not all STSG patients passed the Sharp-Blunt-Distinction test upon measuring points 5 to 9 , which are situated on intact normal skin next to the graft. This finding can be explained by the severity of the initial trauma which may have caused local nerve damage. Alternatively, nerves may have been damaged during operations on the bones and muscles. Results of the 2-Point-Threshold test should be interpreted with caution. Lundborg et al. described problems of 2-PT testing (33). The outcome of this test is highly variable and influenced by the patient's cognitive function, age and by the observer. Some patients do not really discriminate between one or two points but experience a change in the width of the application (34). Many patients in the current study reported that they had difficulties in performing the test and scored 0 points for all measurement points meaning that they could not discriminate between points at all. Although 2-PT testing is one of the most common sensibility tests, it lacks objectivity $(33,34)$. Nearly all the patients of the current study's cohort suffered from severe bone injury and major trauma. Therefore, differences in range of motion of joints, if observed at all, may be explained by underlying bone conditions and not by skin contracture. Eight patients with arthrodesis were not included in the study.

The current study findings with regard to skin elasticity measurements did not meet the authors' expectations. They estimated that groups would differ significantly when comparing the deformation difference (R0) of operated to control site. Lee et al. compared the application of STSG on radial free flap donor sites to a combination of STSG with an acellular dermal matrix. They found STSG alone to be less elastic than normal skin (STSG $0.42 \pm 0.17 \mathrm{~mm}$, control site $0.69 \pm 0.14 \mathrm{~mm} ; p<0.05)$. But the combination of STSG with acellular dermal matrix for radialis free flap donor site did not differ significantly from normal skin $(0.60 \pm 0.15 \mathrm{~mm}$, control site $0.61 \pm 0.16 \mathrm{~mm} ; p=0.22$ ) (35). However, in their study, skin elasticity was measured with the tendon of Musculus flexor carpi radialis beneath the skin, while measurements with underlying tendons and bones were avoided in the current study. Busche et al. measured skin elasticity with Cutometer ${ }^{\circledR}$ on burn scars which were treated conservatively, with STSG alone or STSG in combination with Matriderm ${ }^{\circledR}$. They found a significantly higher loss of elasticity in the STSG group compared to the conservatively treated group and furthermore, incomplete recovery in the conservative group meaning that this group did not regain the elasticity of normal skin (conservative group: $0.83 \pm 0.56 \mathrm{~mm}$, control site $1.16 \pm 0.54 \mathrm{~mm}$; STSG group: $0.7 \pm 0.7 \mathrm{~mm}$, control site:
$0.99 \pm 0.7 \mathrm{~mm}$ ) (27). Both studies compared the scar centre to the healthy skin adjacent which resembles the current study's absolute data measurement point 1 compared to measurement points 6 to 9 as shown in Table V. The current investigators found similar results for the STSG group: The centre of the scar was less elastic than the normal adjacent skin $(0.98 \pm 0.38$ $\mathrm{mm}, 95 \% \mathrm{CI}=0.82-1.14$, range $=0.50-1.71$, vs. $1.20 \pm 0.32 \mathrm{~mm}$, $95 \% \mathrm{CI}=1.07-1.33$, range $=0.71-2.01 \mathrm{~mm} ; p=0.0062)$. In the SEMI group, scar centre and healthy skin were nearly equally elastic $(1.29 \pm 0.39 \mathrm{~mm}, 95 \% \mathrm{CI}=1.15-1.45$, range $=0.54-2.00$, vs. $1.23 \pm 0.27 \mathrm{~mm}, 5 \% \mathrm{CI}=1.13-1.34$, range $=0.60-1.75$; $p=0.8509$ ). In contrast to the conservative treated patients in the Busche's study who suffered superficial dermal burns, the current study's SEMI group did not experience burns and maintained intact full-thickness skin adjacent the operation wound. Sín et al. claimed that the dermal component is very important for reconstruction of a pliable and durable skin (36). In agreement with Sín, the current authors found that areas without dermis (measurement points 1 to 5 in STSG scars) were significantly less pliable than the same area in SEMI patients, when the raw data were analysed. Outside the scar and on the healthy control site, where the skin provides an intact dermal layer, no differences between groups were found.

In some patients, measurement of skin elasticity was difficult. In one STSG patient, the grafted area was too small to be measured. The head of the probe, with a cross-section of $24 \mathrm{~mm}$, exceeded the scar area. In other patients, scar areas were small, so measurements halfway between scar centre and scar margins were performed right next to each other. In contrast, in other patients, scar areas were extremely large with many different underlying tissues which influenced skin elasticity. Some measurements had to be repeated because the probe aperture did not seal properly over the measured points, resulting in air leaks. One elderly female patient had received a SEMI on the palmar aspect of the wrist. Skin elasticity measurement could not be performed on her since the skin deformation exceeded $3 \mathrm{~mm}$ which is the maximum deformation measured by the Cutometer ${ }^{\circledR}$ MPA 580. One patient was excluded from the analysis of skin elasticity because he had received a gastrocnemius flap under the STSG. The muscle flap results in extra tissue at this location and therefore its skin elasticity is affected. In the current study, patients' measurement areas and control sites were not routinely shaved, unlike what Klosova et al. did in their study using the Cutometer (37). Therefore, it is possible that hairs may have affected the light measuring system.

The current study had certain limitations: The patients examined in the study were heterogeneous with regard to age, health status and the surgical procedure performed (STSG or SEMI). Moreover, many studies report skin elasticity to be influenced by age, sex, skin thickness and sun 
exposition (38-41), and skin thickness and sensitivity to decrease with age (42-44).

\section{Complications}

In this retrospective analysis, the current authors revealed a tendency for STSG procedures to have a higher complication rate than SEMI procedures, with more tissue necrosis, impaired wound healing and/or wound dehiscence, secondary tissue granulation and infection. However, the differences between study groups were not statistically significant. This comparative analysis needs further investigation to establish whether a true difference in outcomes exists.

\section{Conclusion}

SEMI is superior to STSGs with regard to skin thickness, sensation, elasticity and a subjective assessment by patients and assessors. As an awareness of health-related quality-oflife increases and economic factors gain importance, the current authors propose the SEMI technique to be a valuable alternative to STSGs for closure of deep soft tissue defects.

\section{Acknowledgements}

This study was financially supported by the KlinStrucMed program of the independent Else-Kröner-Foundation (Else-Kröner-Stiftung) as part of the work towards a Clinical MD degree for JK.

\section{References}

1 Blume PA, Key JJ, Thakor P, Thakor S and Sumpio B: Retrospective evaluation of clinical outcomes in subjects with split-thickness skin graft: Comparing V.A.C. therapy and conventional therapy in foot and ankle reconstructive surgeries. Int Wound J 7(6): 480-487, 2010.

2 Burnett LN, Carr E, Tapp D, Raffin Bouchal S, Horch JD, Biernaskie J and Gabriel V: Patient experiences living with split thickness skin grafts. Burns 40(6): 1097-1105, 2014.

3 Min JH, Yun IS, Lew DH, Roh TS and Lee WJ: The use of Matriderm and autologous skin graft in the treatment of full thickness skin defects. Arch Plast Surg 41(4): 330-336, 2014.

4 Eid A, Elsoufy M, Eid A and Elsoufy M: Shoelace Wound Closure for the Management of Fracture-Related Fasciotomy Wounds. ISRN Orthop 2012: 1-6, 2012.

5 Haslik W, Kamolz LP, Manna F, Hladik M, Rath T and Frey M: Management of full-thickness skin defects in the hand and wrist region: first long-term experiences with the dermal matrix Matriderm ${ }^{\circledR}$. J Plast Reconstr Aesthetic Surg 63(2): 360-364, 2010 .

6 Barnea Y, Gur E, Amir A, Leshem D, Zaretski A, Miller E, Shafir R and Weiss J: Delayed primary closure of fasciotomy wounds with Wisebands ${ }^{\circledR}$, a skin- and soft tissue-stretch device. Injury 37(6): 561-566, 2006.

7 Fitzgerald A, Gaston P, Wilson Y, Quaba A and McQueen M: Long-term sequelae of fasciotomy wounds. Br J Plast Surg 53(8): 690-693, 2000.
8 Gaulke R: Skin extension by multiple incisions. In Vivo 33(2): 495-500, 2019.

9 McBride C, Kempf M, Kimble R and Stockton K: Variability in split-thickness skin graft depth when using an air-powered dermatome: A paediatric cohort study. Burns 43(7): 1552-1560, 2017.

10 Andreassi A, Bilenchi R, Biagioli $M$ and D'Aniello C: Classification and pathophysiology of skin grafts. Clin Dermatol 23(4): 332-337, 2005.

11 Tanner JC, Vandeput J and Olley JF: The Mesh Skin Graft. Plast Reconstr Surg 34(3): 287-292, 1964.

12 Draaijers LJ, Tempelman FRH, Botman YAM, Tuinebreijer WE, Middelkoop E, Kreis RW and Van Zuijlen PM: The Patient and Observer Scar Assessment Scale: A reliable and feasible tool for scar evaluation. Plast Reconstr Surg 113(7): 1960-1965, 2004.

13 Mundy LR, Miller HC, Klassen AF, Cano SJ and Pusic AL: Patient-Reported Outcome Instruments for Surgical and Traumatic Scars: A Systematic Review of their Development, Content, and Psychometric Validation. Aesthetic Plast Surg 40(5): 792-800, 2016.

14 Short Manual Wound QoL, 2015. Available from: https:// www.wound-qol.com/wp-content/uploads/Wound-QoLShortManual-2018-05.pdf

15 Augustin M, Herberger K, Rustenbach SJ, Schäfer I, Zschocke I and Blome C: Quality of life evaluation in wounds: Validation of the Freiburg Life Quality Assessment-wound module, a disease-specific instrument. Int Wound J 7(6): 493-501, 2010.

16 Price $\mathrm{P}$ and Harding K: Cardiff wound impact schedule: The development of a condition-specific questionnaire to assess health-related quality of life in patients with chronic wounds of the lower limb. Int Wound J 1(1): 10-17, 2004.

17 Engelhardt M, Spech E, Diener H, Faller H, Augustin M and Debus ES: Validation of the disease-specific quality of life Wuerzburg Wound Score in patients with chronic leg ulcer. Vasa 43(5): 372-379, 2014.

18 Blome C, Baade K, Debus ES, Price P and Augustin M: The "Wound-QoL": A short questionnaire measuring quality of life in patients with chronic wounds based on three established diseasespecific instruments. Wound Repair Regen 22(4): 504-514, 2014.

19 Finlay AY and Khan GK: Dermatology Life Quality Index (DLQI) - A simple practical measure for routine clinical use. Clin Exp Dermatol 19(3): 210-216, 1994.

20 Basra MKA, Fenech R, Gatt RM, Salek MS and Finlay AY: The Dermatology Life Quality Index 1994-2007: A comprehensive review of validation data and clinical results. $\mathrm{Br} \mathrm{J}$ Dermatol 159(5): 997-1035, 2008.

21 Vercelli S, Ferriero G, Sartorio F, Cisari C and Bravini E: Clinimetric properties and clinical utility in rehabilitation of postsurgical scar rating scales. Int J Rehabil Res 38(4): 279-286, 2015.

22 Reinholz M, Poetschke J, Schwaiger H, Epple A, Ruzicka T and Gauglitz GG: The dermatology life quality index as a means to assess life quality in patients with different scar types. J Eur Acad Dermatology Venereol 29(11): 2112-2119, 2015.

23 Ware JE and Sherbourne CD: The MOS 36-Item Short-Form Health Survey (SF-36). Med Care 30: 473-483, 1992.

24 Wann-Hansson C, Hallberg IR, Risberg B and Klevsgård R: A comparison of the Nottingham Health Profile and Short Form 36 Health Survey in patients with chronic lower limb ischaemia in a longitudinal perspective. Health Qual Life Outcomes 2: 9, 2014. 
25 Van De Kar AL, Corion LUM, Smeulders MJC, Draaijers LJ, Van Der Horst CMAM and Van Zuijlen PM: Reliable and feasible evaluation of linear scars by the patient and observer scar assessment scale. Plast Reconstr Surg 116(2): 514-522, 2015.

26 Troung PT, Lee JC, Soer B, Gaulk CA and Olivotto IA: Reliability and Validity Testing of the Patient and Observer Scar Assessment Scale in Evaluating Linear Scars after Breast Cancer Surgery. Plastic Reconstr Surg 119(2): 487-494, 2007.

27 Busche MN, Thraen A-CJ, Gohritz A, Rennekampff H-O and Vogt PM: Burn Scar Evaluation Using the Cutometer(R) MPA 580 in Comparison to "Patient and Observer Scar Assessment Scale" and "Vancouver Scar Scale". J Burn Care Res 39(4): 516526, 2017.

28 Van Loey NEE, Bremer M, Faber AW, Middelkoop E and Nieuwenhuis MK: Itching following burns: Epidemiology and predictors. Br J Dermatol 158(1): 95-100, 2004.

29 Carrougher GJ, Martinez EM, McMullen KS, Fauerbach JA, Holavanahalli RK, Herndon DN, Wiechman SA, Engrav LH ans Gibran NS: Pruritus in adult burn survivors: Postburn prevalence and risk factors associated with increased intensity. J Burn Care Res 34(1): 94-101, 2013.

30 Poetschke J, Dornseifer U, Clementoni MT, Reinholz M, Schwaiger H, Steckmeier S, Ruzicka $\mathrm{T}$ and Gauglitz GG: Ultrapulsed fractional ablative carbon dioxide laser treatment of hypertrophic burn scars: evaluation of an in-patient controlled, standardized treatment approach. Lasers Med Sci 32(5): 10311040, 2017.

31 Augustin M, Conde Montero E, Zander N, Baade K, Herberger K, Debus ES, Diener H, Neubert T and Blome C: Validity and feasibility of the wound-QoL questionnaire on health-related quality of life in chronic wounds. Wound Repair Regen 25(5): 852-857, 2017.

32 Salomé GM, Blanes L and Ferreira LM: The impact of skin grafting on the quality of life and self-esteem of patients with venous leg ulcers. World J Surg 38(1): 233-240, 2014.

33 Lundborg G and Rosén B: The two-point discrimination test Time for a re-appraisal? J Hand Surg Am 29(5): 418-422, 2004.

34 Craig JC and Johnson KO: The two-point threshold: Not a measure of tactile spatial resolution. Curr Dir Psychol Sci 9(1): 29-32, 2000.
35 Lee JH, Alrashdan MS, Kim SG, Rim JS, Jabaiti S, Kim MJ and Kim SM: Functional and esthetic assessment of radial forearm flap donor site repaired with split thickness skin graft. Eur Arch Oto-Rhino-Laryngology 268(1): 109-115, 2011.

36 Šín P, Stupka I and Brychta P: Evaluation and comparison of composite and split-thickness skin grafts using cutometer MPA 580. Ann Burns Fire Disasters 23(4): 208-213, 2010.

37 Klosová H, Štětinský J, Bryjová I, Hledík S and Klein L: Objective evaluation of the effect of autologous platelet concentrate on post-operative scarring in deep burns. Burns 39(6): 1263-1276, 2013.

38 Dobrev H: Study of human skin mechanical properties by mean of Cutometer. Folia Med 44(3): 120-122, 2002.

39 Ahn S, Kim S, Lee H, Moon S and Chang I: Correlation between a Cutometer and quantitative evaluation using Moire topography in age-related skin elasticity. Ski Res Technol 13(3): 280-284, 2007.

40 Draaijers LJ, Botman YAM, Tempelman FRH, Kreis RW, Middelkoop E and Van Zuijlen PM: Skin elasticity meter or subjective evaluation in scars: A reliability assessment. Burns 30(2): 109-114, 2004.

41 Enomoto DNH, Mekkes JR, Bossuyt PMM, Hoekzema R and Bos JD: Quantification of cutaneous sclerosis with a skin elasticity meter in patients with generalized scleroderma. J Am Acad Dermatol 35(3): 381-387, 1996.

42 Pawlaczyk M, Lelonkiewicz $M$ and Wieczorowski M: Agedependent biomechanical properties of the skin. Postepy Dermal Alergol 30(5): 302-306, 2013.

43 Takema Y, Yorimoto Y, Kawai M and Imokawa G: Age-related changes in the elastic properties and thickness of human facial skin. Br J Dermatol 131(5): 641-648, 1994.

44 Louis DS, Greene TL, Jacobson KE, Rasmussen C, Kolowich P and Goldstein SA: Evaluation of normal values for stationary and moving two-point discrimination in the hand. J Hand Surg Am 9(4): 552-555, 1984. 